\title{
Association between Denture Use, Chewing Ability, and Cognitive Function Using the Korean Longitudinal Study of Aging (2006-2018)
}

\author{
Se-Rim Jo', Jae-Hyun Kim², Jong-Hwa Jang ${ }^{1,3}$ \\ ${ }^{1}$ Department of Public Health, Graduate School, Dankook University, Korea \\ ${ }^{2}$ Department of Health Administration, Dankook University College of Health Science, Korea \\ ${ }^{3}$ Department of Dental Hygiene, College of Public Health Sciences, Dankook University, Korea
}

Objective: The chewing function is the first step in the process of digestion food and is a very important factor in maintaining and improving the state of general health. This study aimed to investigate the relationship between denture use, masticatory ability and cognitive function.

Methods: The participants were 9,998 people born before 1963, preconstructed from the $7^{\text {th }}$ special survey data of the 2019 Aging Research Panel using the GEE model. In accordance with the KLoSA study protocol, trained investigators obtained consent from participants and conducted face-to-face interviews using a computer-based personal interview program. Independent variables, whether or not dentures were used, were measured for mastication discomfort. As control variables, 5 items of demographic characteristics (age, education level, gender, marital status, activity restriction) and 3 items of health status and behavioral factors (drinking, health insurance type, number of diseases) were included as covariates. Cognitive function (MMSE), an outcome variable, consists of a total of 12 items and was judged on a total of 30 points. Item contents include time orientation ( 5 points), place orientation (5 points), memory recall ( 3 points), memory registration (3 points), language function ( 7 points), attention and calculation (5 points), understanding and A judgment ( 2 points) area was investigated. A score of 27 or higher was considered normal, a score of 23-26 was considered mild cognitive impairment, a score of 22 or lower was considered moderate cognitive impairment, and a score of less than 20 was considered dementia. Generalized Estimating Equation (GEE) was analyzed using SAS software.

Results: The number of subjects wearing dentures was 2.402 (24.0\%), and the average cognitive function was 25.44 points, indicating mild cognitive impairment. Cognitive function was lower in denture wearers (22.96 points) than non-denture wearers (26.22 points) $(\mathrm{p}<0.001)$, and the lowest in those who had no masticatory ability ( 18.5 points $)(p<0.001)$. Cognitive functions is related to the elderly, elementary school graduates (vs. $\geq$ college), females (vs. male), divorce and separation (vs. married), activity restrictions (vs. non-working restriction), medical care (vs. National Health Insurance), it was lower in non-drinkers (vs. alcohol drinkers) $(\mathrm{p}<0.001)$. When the general characteristics were controlled and dentures were worn, the cognitive function was significantly lower when the masticating function was uncomfortable $(\mathrm{p}<0.001)$.

Conclusions: In summary, the greater the denture use and mastication discomfort, the lower the cognitive function. Therefore, it is considered that it can contribute to improving cognitive function through oral health promotion by reducing the complaints of mastication discomfort, and it is suggested that it should be considered in dementia prevention programs for improving cognitive function.

Keywords: Chewing ability, Cognitive function, Dental health, Denture

Copyright (C) 2021. Korean Academy of Preventive Dentistry. All rights reserved.

This is an Open Access article distributed under the terms of the Creative Commons Attribution Non-Commercial License (http://creativecommons.org/licenses/ by-nc/4.0) which permits unrestricted non-commercial use, distribution, and reproduction in any medium, provided the original work is properly cited. 